\title{
THE INFLUENCE OF TERNARY ADDITIONS ON THE TRANSFORMATION TEMPERATURES OF NiTi SHAPE MEMORY ALLOYS - A THEORETICAL APPROACH
}

\author{
P.M. HUISMAN-KLEINHERENBRINK and J. BEYER \\ University of Twente, Faculty of Mechanical Engineering, Laboratory for Materials Science, PO Box \\ 217, NL-7500 AE Enschede, The Netherlands
}

\begin{abstract}
A newly developed model, predicting the transformation temperatures of NiTi-based ternary alloys as a function of composition, is shortly described. The model is discussed with respect to the class of alloy systems it is applicable to.
\end{abstract}

\section{1.-Introduction.}

Each application of Shape Memory Alloys requires its own specific set of transformation temperatures. These transformation temperatures depend on the thermomechanical history and the composition of the alloy. The influence of ternary additions to NiTi on its transformation temperatures is most frequently empirically determined for a number of transition elements (e.g. $/ 1 /$ ). However, because of the innumerable amount of possibilities, it is wished to have a more fundamental understanding of the influence of ternary additions to NiTi on the transformation temperatures.

Recently, we developed a new, semi-empirical model, predicting the transformation temperatures of a ternary $\mathrm{T} i$-based alloy system as a function of the composition. This model is extensively described elsewhere /2,3/. For this paper, it is our aim to give an overview of class of alloy systems, to which this model can be applied, and the limitations of the model.

\section{2. - Survey of literature.}

There is general agreement on the electronic character of the transformation, as first proposed by Wang /4/. A survey of literature $/ 1,4-8 /$, presented elsewhere $/ 2,3 /$, led to the conclusion that there are three important quantities determining the transformation and the corresponding transformation temperatures, i.e.

1) the Fermi energy, $E_{F}$

2) the real number of valence d-electrons, $n_{d}$ (not the number taken from the periodic system)

3) the partial density of valence d-states, d-DOS

It is remarkable that in most models, temperature is not involved. For example, Fermi Surface nesting models consider the Fermi Surface at zero Kelvin. There is, however, not yet a simple model to predict the transformation temperatures as a function of the composition.

\section{3.-The development of a new model}

Because no simple model, predicting the transformation temperatures as a function of 
the composition, is available, we developed a new model, in which the above three quantities, the influence of ternary additions on these quantities and the temperature are involved. The model so developed is based on the hypothesis that there are two special states $\left(A_{1}\right.$ and $\left.A_{2}\right)$, both with energy $E_{A}$ but with different $k$-vectors or spin, of which the occupation probability $\dot{\rho}$ should reach a (composition independent) critical value $\rho *$ for transformation to take place. States $A_{1}$ and $A_{2}$ and their energy $E_{\mathbf{A}}$ are unknown, so far. For a ternary, $E_{\mathbf{A}}$ can be interpolated between the values of $E_{A, N i T i}$ and $E_{A, M x T}$, which can in turn be calculated from the transformation temperature of $\mathrm{NiTi}$ and $\mathrm{MxTi}$.

Assuming that the Fermi-Dirac distribution function also accounts for alloys, the critical occupation probability $\rho^{*}$ is written as

$$
\rho *=\frac{1}{\exp \left[\frac{E_{A}-E_{F}}{k_{B} T_{0}}\right]+1}
$$

with $k_{B}$ Boltzmann's constant and $T_{0}$ the temperature at which the austenite and the martensite are in thermodynamic equilibrium. So,

$T_{0}=\frac{-1}{k_{B} \ln \left[1 / \rho^{*}-1\right]}\left(E_{F}-E_{A}\right)=C\left(E_{F}-E_{A}\right)$

It is shown $/ 2,3 /$ that, in a simplest approximation, $\rho *=0.5 \sqrt{2} \cong 0.71$ or $\rho^{*}=1-0.5 \sqrt{ } 2 \cong 0.29$, depending on whether $E_{A}<E_{F}$ or $E_{A}>E_{F}$, which is not clear yet. The value of $\mathrm{C}$, related to $\rho^{*}$, is indicated in table 1 . It is noted that the values of $\rho^{*}$ will be further indicated with 0.71 and 0.29 , but the exact values $0.5 \sqrt{ } 2$ and $1-0.5 \sqrt{ } 2$ are used in the presented calculations.

Table 1: values of $\rho^{*}$ and $\mathrm{C}$
\begin{tabular}{|r|c|r|}
\hline$\rho^{*}$ & $\mathrm{C}(\mathrm{K} / \mathrm{eV})$ & $\mathrm{k}_{\mathrm{B}} \mathrm{C}$ \\
\hline $0.5 \sqrt{ } 2$ & $1.3166 * 10^{4}$ & 1.13 \\
$1-0.5 \sqrt{ } 2$ & $-1.3166 * 10^{4}$ & -1.13 \\
\hline
\end{tabular}

Because values of $T_{0}$ are unknown, the assumption is made that the difference $T_{0}-M_{S}$ varies linearly with the composition. The model is further developed to give $M_{s}$ as a function of the concentration $x$ of the element 'Mx' in the NiTi-MxTi $\left(\mathrm{Ni}_{0.50-\mathrm{x}} \mathrm{T} \mathrm{i}_{0.50} \mathrm{Mx}_{\mathrm{x}}\right)$ alloy system. The element $\mathrm{Mx}$ should be an element which itself forms a $\mathrm{B} 2$ alloy with $\mathrm{Ti}(\mathrm{Mx}=\mathrm{Fe}$, Co, $\mathrm{Tc}$, Ru, Rh, Pd, Os, Ir, Pt, Au). In that case, the d-DOS of NiTi and MxTi are similar, so that simple interpolations between the d-DOS of NiTi and MxTi are allowed. This development is described elsewhere $12,3 /$. One of the approximations made in the development of this model is given by the description of the number of valence d-electrons of the ternary alloy, i.e.

$n_{d, N i T i N X}(x)=n_{d, N i T i}+2 x\left(n_{d, N x T i}-n_{d, N i T i}\right)+\alpha 2 x(2 x-1)$

in which $\alpha$ is a fit parameter. Parameter $\alpha$ represents the exchange of electrons between $\mathrm{s}-, \mathrm{p}$ - and $\mathrm{d}$-bands and between the atoms in the alloy. The value of $\alpha$ should be such that (with $n_{t}=$ the total number of valence electrons) $n_{t}-2<n_{d}<n_{t}$ for any $0.00<x<0.50$. Use of this parameter, together with the use of the $M_{s}$ temperature of $\mathrm{NiTi}$ and MxTi makes the model into a semi-empirical model. Further development of the model to quaternary alloys is possible $/ 2,3 /$.

\section{4. - Results and discussion.}

As input parameters, we used $E_{F}, n_{d}$ and the d-DOS, as calculated with the Augmented Spherical Wave method by Slavenburg and Inglesfield /9/. The experimental data are taken from $/ 1,2,10,11 /$. Results are given in figure 1 . The values of $\rho *(0.71$ and 0.29 ) are indicated in the plots (solid curves). The dashed curves in figure 1 
NiTiPd

(ASW)

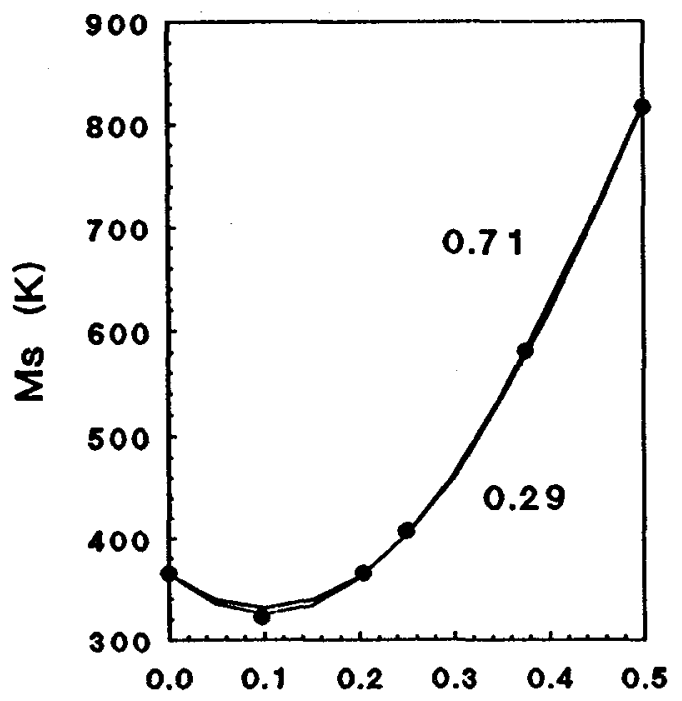

at. fraction $\mathrm{Pd}$

NiTiAu

(ASW)

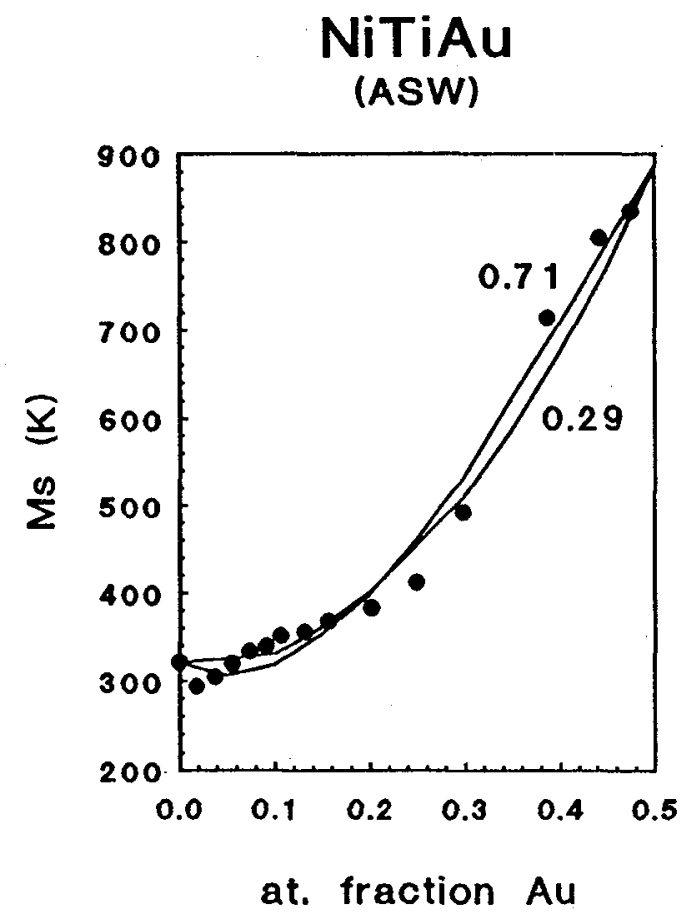

at. fraction $\mathrm{Au}$
NiTiPt

(ASW)

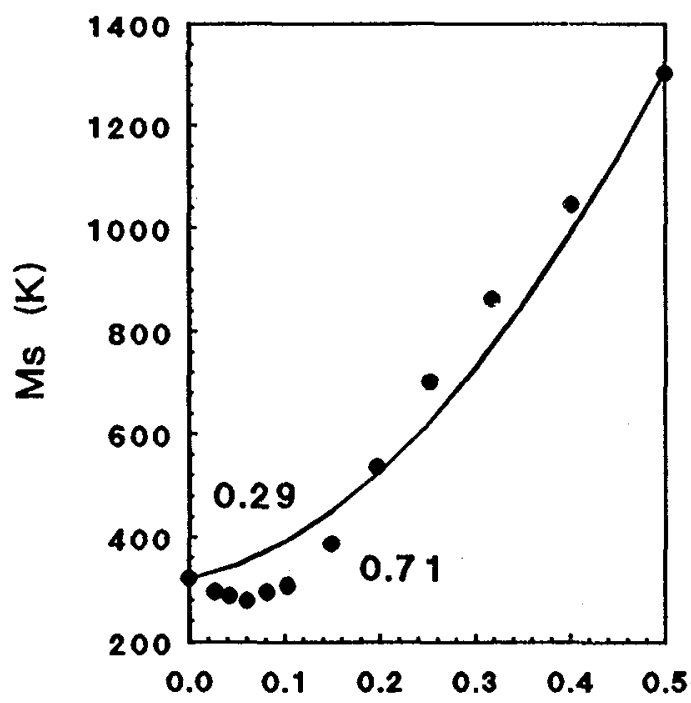

at. fraction $\mathrm{Pt}$

Figure 1.- $\mathrm{M}_{\mathrm{s}}$ as a function of composition. - : calculations; - : empirical data, as given in 1iterature (see text). 

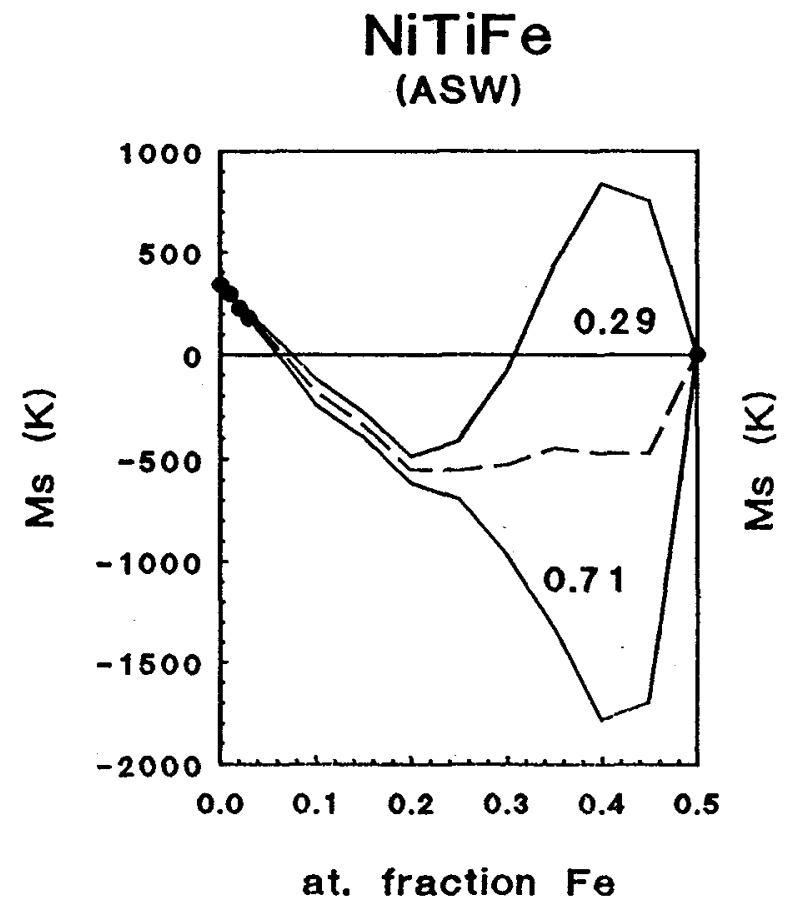

NiTiCo

(ASW)

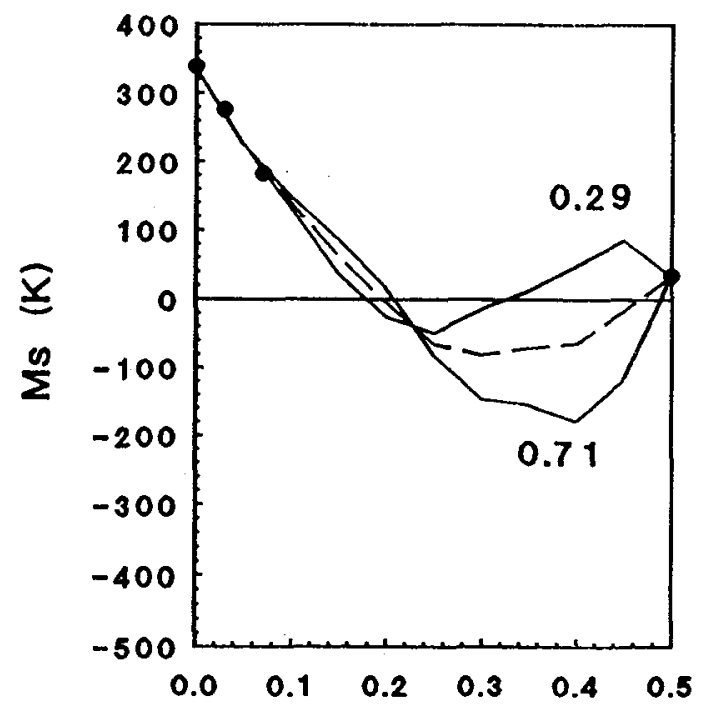

at. fraction Co
FeTiPd

(ASW)

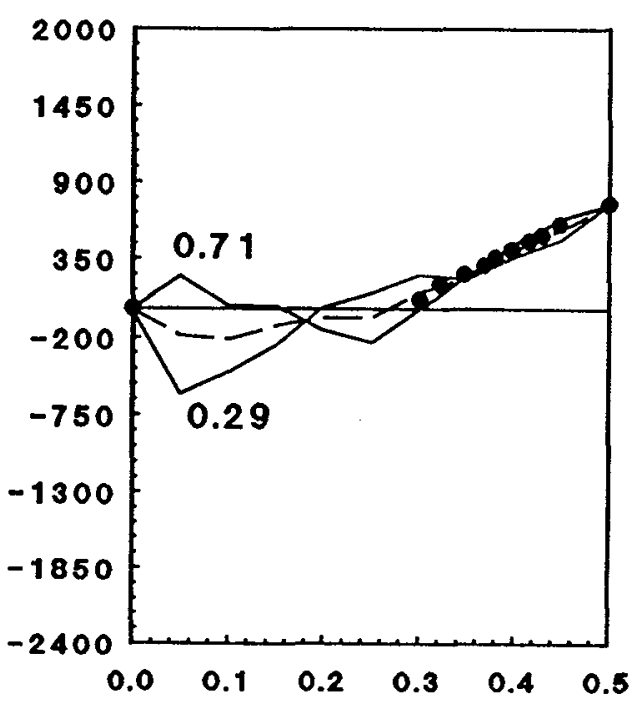

at. fraction $\mathrm{Pd}$

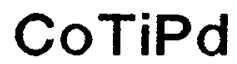

(ASW)

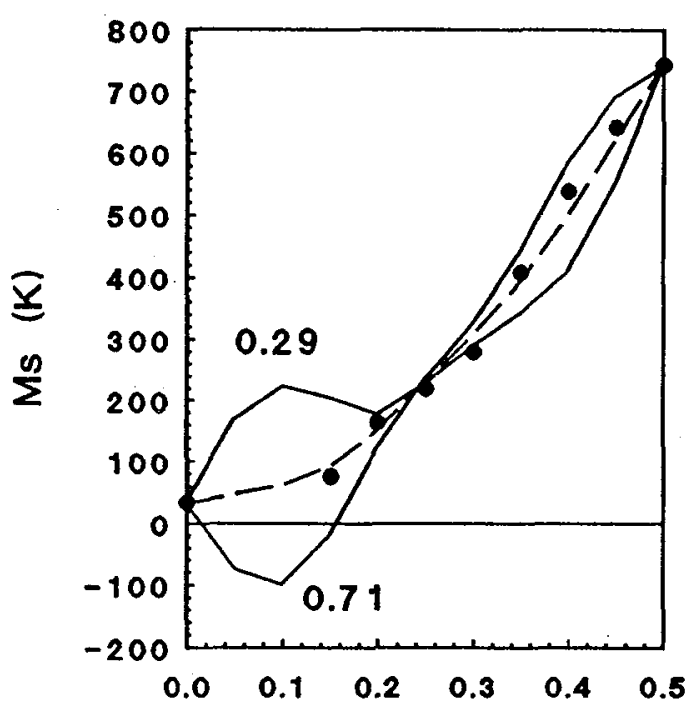

at. fraction $\mathrm{Pd}$ 
describe the average of the curves for $\rho^{*}=0.71$ and 0.29 . The corresponding values of $\alpha$ are given in table 2. In all cases, the value of $\alpha$ is such that $n_{t}-2<n_{d}<n_{t}$.

Table 2: values of $\alpha$

\begin{tabular}{|c|c|c|}
\hline alloy & $\alpha\left(\rho^{*}=0.29\right)$ & $\alpha\left(\rho^{*}=0.71\right)$ \\
\hline NiTiFe & -0.202 & 0.226 \\
NiTiCo & -0.148 & 0.010 \\
FeTiPd & -0.029 & 0.170 \\
CoTiPd & -0.115 & -0.004 \\
NiTiPd & -0.055 & 0.083 \\
NiTiPt & -0.080 & 0.056 \\
NiTiAu & -0.008 & 0.107 \\
\hline
\end{tabular}

Ti-based ternary $B 2$ alloy systems

The calculated curve for NiTiPd describes the experimentally found minimum in the $M_{s}-x$ curve very well. This is the first theory accurately describing this minimum, as far as we know.

The fits for FeTiPd and CoTiPd show that the model also accounts for other Ti-based B2 alloys than the NiTi-based.

In some cases, the average curves describe the experimental data points better than the curves for either value of $\rho^{*}$. It is explained in $/ 2,3 /$ that this is due to a combined effect of the accuracy of the d-DOS, used as an input, and some approximations, made in the development of the model, $i . e$. the linear interpolations between the d-DOS of NiTi and MxTi to determine the Fermi level and the energy of states $A_{1}$ and $A_{2}$ in the ternary alloys $/ 2,3 /$. So, the applicability of the newly developed model in the present formulation is restricted by the accuracy of the input and the validity of the approximations for the alloy system under consideration. These aspects, however, do not principally affect the applicability of the model.

Another effect of the approximations made is shown in the fits for NiTiPt and NiTiAu. The second order approximation for $n_{d, \text { NiTiMx }}(x)$ is not thought to be sufficiently accurate for these alloy systems, because for $P t$ and $A u$, also $4 f$ electrons play a role in the exchange of electrons between the bands. A higher order approximation is required in these cases. (Note that $\mathrm{Pt}$ and $\mathrm{Au}$ are the only sixth period elements considered.)

Other transition elements

Transition elements, other than those which form a B2 alloy with Ti, can also be handled with the model, as shown for $\mathrm{Zr}$ and $\mathrm{Mn} / 2,12,13 /$. The $\mathrm{E}_{F}, \mathrm{n}_{\mathrm{d}}$ and $\mathrm{d}-\mathrm{DOS}$ can, in principal, be calculated, assuming a hypothetical B2 structure for MxTi. A practical problem in this case is that the transformation temperatures of the extreme binary alloy MxTi is unknown, since this alloy does not have a B2 structure, and thus cannot transform from a B2 structure to an orthorhombic structure. Combination of empirical data of different, well chosen alloy systems, can be used to eliminate the unknown terms from the equations.

\section{Other elements}

When other than the transition elements are added to the NiTi, the principles of the model should still be valid. However, it is very well possible that approximations, made during the development of the model, are not valid in these cases. For example, simple linear interpolations of the d-DOS cannot be made when the d-DOS of the extreme binary alloys NiTi and MxTi are not similar. Especially when the number of available d-states in MxTi is less than the number of electrons in a corresponding ternary alloy, problems arise in one step of the calculation procedure. (Note that the average number of available valence d-states per atom is constant when only transition elements are involved.) 
The model can also be applied to non-stoichiometric alloys, considering structural vacancies as a ternary addition. However, the present formulation of the model is not suitable for this, because in the present formulation, an intermediate step exists in which the number of electrons is larger than the number of available electron states $/ 2 /$. To handle these vacancies, the model should be adapted. Consideration of structural vacancies as a third element is shows co be possible $12,12 /$.

\section{Other alloy systems}

There are no principle arguments that prevent application of the newly developed mode1 to Cu-based of Fe-based alloy systems. However, the role of states $A_{1}$ and $A_{2}$ may very well be different in these cases. There may be more than two states playing a role. It is also possible that these states do not have the same energy. When these states are known, the value of $\rho^{*}$ can be determined very easily.

Other diffusionless transformations

The model is not principally restricted to ternary systems. It can also be applied to e.g. the binary Ti-Zr system. In that case, the transformation temperature, the Fermi energy, the number of valence d-electrons and the $d-D O S$ of pure $T i$ and $Z r$ should be used as an input.

\section{5. - Conclusions}

The recently developed model is thought to be applicable to principally all diffusionless transformations. In the present formulation, its use is restricted to those alloy systems, for which the simple approximations, made during the development of the model, are valid.

\section{Acknowledgements}

This work is a part of the Ph.D. work, carried out at the University of Twente. The Foundation for Advanced Metals Science (Stichting Geavanceerde Metaalkunde, SGM) is gratefully acknowledged for financial support. Prof. B.H. Kolster, Prof. A. van Silfhout and Dr. J. Mass are acknowledged for stimulating discussions. Prof. J.E. Inglesfield and Drs. P. Slavenburg are thanked for calculations of, and discussion about, the input parameters of the model.

\section{References}

11/ HONMA $T$, MATSUMOTO $M$, SHUGO $\mathrm{X}$, NISHIDA M, YAMAZAKI I Proc. of the Fourth Int. Conference on Titanium, Kyoto, Japan, May 19-22 1980

12/ HUISMAN-KLEINHERENBRINK PM, Ph.D. thesis, University of Twente, Enschede, the Netherlands 1991

13/ HUISMAN-KLEINHERENBRINK PM, MAAS JH, BEYER $\mathrm{J}$, to be submitted

$14 /$ e.g. WANG FE, DESAVAGE BF, BUEHLER WJ, HOSLER WR, J. Appl. Phys. 39 (1968) 2166

/5/ MITCHELL MA, WANG FE, CULLEN JR J. Appl. Phys. 45 91974) 3337

$16 /$ e.g. SHABALOVSKAYA SA Phys. Stat. Sol. (B) 132 (1985) 327

/7/ e.g. ZHAO G.-L, LEUING TC, HARMON BN, KEIL M, MUELLNER M, WEBER W, Phys. Rev. B 40 (1989) 7999

/8/ EGORUSHKIN VE, KUL'MENT'YEV AI, FLAT A YA, J of Phys. F: Met. Phys. 17 (1987) 289 and references therein

19/ SLAVENBURG P, INGLESFIELD JE, private communication

110/ VORONIN VP, KHACHIN VN, Fiz. Met. i. Metalloved. 68 (1989) 526

/II/ KHAGHIN VN, MATVEEVA NM, SIVOKHA VP, CHERNOV DB, KOVNERISTYI YU K, Dokl. akad. nauk. SSSR 257 (1981) 167

112/ HUISMAN-KLEINHERENBRINK PM, VAN ROSSUM LJ, BEYER J, to be submitted

/13/ HUISMAN-KLEINHERENBRINK PM, YIN JIAN, BEYER J, Materials Letters 11 (1991) $145-150$ 